\title{
Pénuries de médicaments en établissement de santé : une étude qualitative à partir de cas réels pris en charge par les pharmaciens hospitaliers
}

\author{
par Marine Floutier, Suzanne Atkinson, Stéphane Roux et Jean-François Bussières
}

J. Can. Pharm. Hosp. 2021;74(3):277-81

DOI: 10.4212/cjhp.v74i3.3155

\section{INTRODUCTION}

En 2019, l’Association des pharmaciens du Canada a noté que " les pénuries de médicaments continuent dêtre une source de vive inquiétude qui ne cesse de samplifier au sein du système canadien de soins de santé. La gestion des problèmes d'approvisionnement en médicaments est devenue une activité malvenue dans lexercice quotidien de la profession de pharmacien; elle monopolise du temps et des ressources qui ne peuvent être consacrés à la prestation de soins directs ${ }^{1}$.

Dans une enquête menée en 2018 auprès de 1500 citoyens canadiens, on a observé qu'un Canadien sur quatre a été touché personnellement par une pénurie de médicaments au cours des trois dernières années ou connaît quelqu'un qui l'a été ${ }^{2}$ Dans une autre enquête menée en 2018 auprès des pharmaciens, on a remarqué que les pénuries de médicaments ont augmenté, voire beaucoup augmenté, au cours des trois à cinq dernières années, d’après $79 \%$ des répondants ${ }^{3}$.

Les pénuries de médicaments font partie du quotidien des pharmaciens communautaires et hospitaliers depuis au moins deux décennies ${ }^{4}$. La crise vécue avec Sandoz Canada en 2012 a laissé sa marque et changé les pratiques au sein des établissements de santé $e^{5}$. Au fil des années, le nombre d'épisodes de pénurie n’a fait quaugmenter. Videau et collab. ont mentionné qu'en l'espace de 12 mois, soit du 31 août 2016 au 4 septembre 2017, il y a eu 2129 ruptures d'approvisionnement de différents médicaments au Canada selon le site web canadien consacré à la déclaration obligatoire des ruptures de fourniture de médicaments par les fabricants. Ces interruptions ont duré en moyenne 118 jours (écart-type 113 jours) et concernaient la plupart des classes thérapeutiques ${ }^{6}$. D’autres études ont confirmé l'importance et la croissance du nombre de pénuries de médicaments au Canada ${ }^{7,8}$.

S'il existe plusieurs études permettant détablir le nombre et la variété des médicaments en pénurie au Canada, il existe peu de travaux mettant en évidence les actions mises en place par les pharmaciens hospitaliers pour limiter les impacts de ces pénuries. Nous nous sommes intéressés à décrire ces actions au sein des établissements de santé d'un groupe d'approvisionnement en commun au Canada.

\section{MÉTHODE}

Il s'agit d'une étude descriptive rétrospective.

Lobjectif principal est de décrire et de quantifier l'impact de la gestion des pénuries de médicaments en établissement de santé sur la charge de travail et la complexité des actions pharmaceutiques des pharmaciens hospitaliers d'un même groupe d'approvisionnement en commun (GAC).

$\mathrm{Au}$ Québec, trois GAC assurent la gestion des ententes contractuelles entre les fabricants de médicaments, les grossistes de médicaments et les établissements de santé. Le chef du département de pharmacie de chaque établissement est responsable détablir la liste locale des médicaments disponibles et de mandater les quantités annuelles de médicaments requises pour assurer les soins des patients. L'étude cible le chef du département de pharmacie (ou son représentant) des 12 établissements de santé membres de Sigmasanté (GAC représentant la région de Montréal et de Laval). Létude a été menée en septembre 2019. Les participants ont été avisés que la participation à létude était volontaire et que les résultats agrégés seraient publiés. L'étude cible l'exercice financier du $1^{\text {er }}$ avril 2018 au 31 mars 2019.

À partir d'une séance de remue-méninges sur les ruptures d'approvisionnement les plus importantes vécues au cours des dernières années et d'une revue documentaire sur la gestion des pénuries, nous avons déterminé quatorze variables permettant de qualifier l'organisation, la gestion des stocks, les sources de données consultées et les pratiques d’approvisionnement de chaque établissement. De plus, nous avons établi 26 actions pharmaceutiques liées à la gestion des pénuries.

Afin de faciliter la réflexion des pharmaciens, les investigateurs ont retenu dix médicaments injectables (sauf un) ayant été en pénurie en 2018-2019 soit : alcaloïde de la vinca (c.-à-d. vincristine, vinblastine), bleu de méthylène, céfazoline, cisatracurium, corticostéroides (c.-à-d. hydrocortisone, 
méthylprednisolone, dexaméthasone), dexmédétomidine, érythromycine pommade ophtalmique, leucovorin, chlorure de potassium, succinylcholine. Ces médicaments ont été retenus parce qu'ils représentent diverses classes thérapeutiques et qu'ils ont probablement eu un impact sur la plupart des membres du GAC.

Les membres de l'équipe de recherche (MF, DL, SA, SR, JFB) ont développé un questionnaire sous forme de fichier texte (Word, Microsoft) et l'ont soumis à la discussion. Ce questionnaire suit la chronologie d'une pénurie, de la prise de connaissance de la pénurie jusquà son impact ou non sur le patient, ce qui favorise lévaluation de chaque étape et la détermination de moyens d'atténuation des conséquences à chacune d'elles. Il vise à répertorier et à mettre en évidence l'impact des pénuries sur la charge de travail et sur la complexité des actions pharmaceutiques découlant des pénuries de médicaments. Léquipe de recherche comporte au moins un pharmacien représentant un établissement pour adultes et un établissement pédiatrique. Le questionnaire a été envoyé à chacun des 12 chefs de département de pharmacie des établissements de santé affiliés à Sigmasanté.

Seules des statistiques descriptives ont été effectuées.

\section{RÉSULTATS}

Dix pharmaciens détablissements associés au GAC Sigmasanté ont participé à létude (taux de participation de $83 \%$, $10 / 12)$; ils provenaient de centres hospitaliers universitaires $(n=3)$, d'un institut universitaire $(n=1)$, de centres intégrés universitaires de santé et de services sociaux $(n=5)$ ou de centres intégrés de santé et de services sociaux $(n=1)$.

Les pharmaciens désignés pour répondre au questionnaire soccupaient de la gestion des pénuries de médicaments depuis moins de cinq ans (3/10) ou plus de cinq ans (7/10). Les répondants, tous titres d'emploi confondus, ont estimé le temps moyen par semaine consacré à la gestion des pénuries de médicaments comme étant inférieur à 6 heures $(n=4)$, de 6 à 20 heures $(n=5)$ et de plus de 20 heures $(n=1)$.

Afin de se tenir au courant des pénuries, les répondants consultent la liste des médicaments en rupture de stocks provenant du grossiste Mckesson Canada $(n=10 / 10)$, les listes que fournissent directement les fabricants par courriel $(n=8 / 10)$, des communications par courriel provenant du GAC et de collègues $(n=7 / 10)$ et le site Pénuries de médicaments Canada (www.penuriesdemedicaments.ca) $(n=5 / 10)$.

Les répondants déclarent maintenir un stock de produits pour 10 à 90 jours selon le caractère critique du produit, lespace et les pratiques.

Les répondants nont pas tous été affectés de la même façon par la pénurie des 10 médicaments ciblés dans notre étude : 10 produits $(n=4)$, neuf produits $(n=1)$, huit produits $(n=1)$, sept produits $(n=1)$, six produits $(n=2)$ pour un total de 76 épisodes de pénurie commentés par les répondants.
Les répondants disposent d'une politique et procédure encadrant la gestion des pénuries de médicaments $(n=5)$, discutent en comité des actions à entreprendre $(n=6)$, effectuent des prêts et des emprunts auprès d'autres établissements de santé $(n=7)$ ou des pharmacies d'officine $(n=2)$. Une majorité de répondants $(n=9)$ achètent le maximum des produits qui leur sont alloués lorsque cela est applicable.

Ces 25 actions envisagées ont été mises en place dans $4 \%$ à $83 \%$ des situations durant les 76 épisodes de pénurie. Un seul épisode de pénurie de médicament a été associé à une déclaration d'incident-accident médicamenteux.

Le tableau 1 présente un profil des actions pharmaceutiques liées à la gestion des pénuries de médicaments en établissement de santé. Comme toutes les pénuries n'ont pas forcément affecté chacun des répondants, compte tenu des stocks disponibles et des patientèles traitées, létude met en évidence un total de 76 épisodes de pénurie sur une possibilité de 100 (c.-à-d. 10 médicaments proposés à 10 répondants).

Le tableau 2 présente le profil du degré d'accord des répondants avec les énoncés entourant la gestion des pénuries de médicaments.

\section{DISCUSSION}

Cette enquête menée auprès de pharmaciens hospitaliers d'un groupe d'approvisionnement en commun d'une des principales villes canadiennes (Montréal) met en évidence l'impact des pénuries sur la charge de travail et la complexité des actions pharmaceutiques découlant des pénuries de médicaments.

En ce qui concerne la charge de travail, tous les répondants ont indiqué avoir désigné un pharmacien affecté à la gestion des pénuries des médicaments. De plus, sept des dix répondants y travaillent depuis plus de cinq ans et une majorité consacre plus de six heures par semaine à la gestion de ces ruptures de stocks. Shaban et collab. ont sondé des départements de pharmacie des établissements de santé du programme des vétérans américains ${ }^{9}$. Des 17 répondants, près de la moitié des établissements interrogés ont reconnu avoir mis en place un groupe de travail $(n=8)$ pour gérer les pénuries de médicaments et atténuer leur impact. Claus et collab. ont évalué l'effet des pénuries de médicaments en Belgique et confirmé qu'elles avaient un impact important sur la charge de travail et les coûts d'acquisition ${ }^{10}$. De Weerdt et collab. se sont également intéressés de plus près à la charge de travail des pharmaciens belges exposés à des ruptures d'approvisionnement de médicaments ${ }^{11}$. Les auteurs ont noté que les pharmaciens d'hôpitaux ont consacré une médiane de 109 minutes par semaine aux problèmes d'approvisionnement en médicaments (min. 40; max. 216). Cinquante-neuf pour cent $(59 \%)$ du temps total consacré aux problèmes d'approvisionnement en médicaments relevait des pharmaciens d'hôpitaux, 27 \% des techniciens en pharmacie ; le reste a été effectué par du personnel logistique ou administratif. 
Envoi d'un avis courriel (note) aux pharmaciens $(n=76)$

Envoi d'un avis courriel (note) aux médecins

Consultation des pharmaciens dans les équipes cliniques touchées par la pénurie

Envoi d'un avis courriel (note) aux infirmières

52

Mise en place d'un changement de pratique auprès des prescripteurs afin de réserver les quantités à certaines

49

indications ou pratiques

Utilisation d'un autre format

Tenue d'une ou de plusieurs réunions avec les cliniciens concernés

37

Création d'une nouvelle fiche " produit » dans le logiciel d'approvisionnement

Révision des quotas des produits concernés dans le logiciel des cabinets ou des réserves d'étage (min.-max.) 26

Création d'une nouvelle fiche " produit » dans le logiciel « dossier clinique informatisé »

Révision des quotas des produits concernés dans le logiciel d'approvisionnement (min.-max.)

Utilisation d'une autre molécule

Mise en place d'un changement de pratique auprès des utilisateurs (infirmières) afin de réserver les quantités à

certaines indications ou pratiques

Ajout d'une alerte dans le dossier pharmacologique informatisé

Réemballage / manipulation pour servir d'une façon différente (p. ex. préparation en seringues vs service en fiole)

20

Utilisation d'une autre teneur

Création d'une nouvelle fiche « produit » dans le logiciel de gestion des cabinets

Demande d'importation d'un produit de remplacement au Programme d'accès spécial de Santé Canada

Report d'activités cliniques (p. ex. dose omise, activité médicale reportée)

Modification requise à la pompe d'alimentation parentérale

Modification requise aux plateaux de réanimation

Modification requise aux protocoles (p. ex. feuilles d'ordonnances prérédigées)

Modification requise aux plateaux d'anesthésie

Prolongation de la date de péremption de stocks périmés résiduels

\section{TABLEAU 2. Profil du degré d'accord des répondants aux énoncés entourant la gestion des pénuries de médicaments}

\section{Énoncé}

Les équipes de gestion des départements de pharmacie sont les mieux placées pour planifier les besoins en médicaments et tous les médicaments jugés importants pour la pratique devraient être stockés pour 90 jours ou plus en inventaire de l'établissement

Il est essentiel de préserver le rôle et le pouvoir d'achat des groupes d'approvisionnement en commun au Québec pour assurer la sécurité des stocks de médicaments en établissement de santé

TA $\quad$ PA $\quad$ PD $\quad$ TD $\quad$ PR

La baisse forcée des prix des médicaments génériques n'est pas étrangère à la réduction des marges d'inventaire des fabricants de médicaments génériques au Canada

$\mathrm{TA}=$ totalement en accord, $\mathrm{PA}=$ partiellement en accord, $\mathrm{PD}=$ partiellement en désaccord, $\mathrm{TD}=$ totalement en désaccord, $\mathrm{PR}=$ pas de réponse. 
Environ un tiers du temps total a été consacré à la collecte d'informations sur le problème d'approvisionnement. Deux enquêtes menées auprès de pharmaciens hospitaliers européens ont également confirmé l'impact des pénuries sur la charge de travail des pharmaciens $s^{12,13}$.

En ce qui concerne la complexité des actions pharmaceutiques mises en place, notre étude met en évidence un total de 25 actions pharmaceutiques potentielles, appliquées dans une proportion variant entre $4 \%$ et $83 \%$ selon les dix médicaments ciblés dans notre enquête. Une partie importante de ces actions nest pas visible à l'extérieur du département de pharmacie. Dans de nombreux cas, le patient ne souffre pas du manque de médicament, grâce au pharmacien désigné qui entreprend de nombreuses actions pour trouver des stratégies permettant d'utiliser des quantités résiduelles du médicament en pénurie sur le marché. Dans $49 \%$ des cas, les répondants ont dû demander aux prescripteurs un changement de pratique afin de réserver les quantités nécessaires à certaines indications ou de modifier leur approvisionnement (p. ex. changement de format [49\%], changement de molécule [30\%], changement de teneur [20\%]). Bien que certains de ces changements semblent banals, ils accroissent tous le risque d'incidents et d'accidents. De plus, chaque changement affecte des dizaines de personnes de titres d'emplois différents (p. ex. pharmaciens, assistants techniques en pharmacie, médecins, infirmières, infirmières auxiliaires) qui travaillent dans plusieurs quarts de travail ; il faut souvent de deux à quatre semaines pour réussir à joindre tous les intervenants concernés. Dans un établissement de santé, des milliers de gestes cliniques sont posés chaque jour et de nombreux changements de pratique liés à la gestion des pénuries de médicaments augmentent les risques derreurs médicamenteuses.

Des 25 actions déterminées, cinq sont effectuées dans au moins $45 \%$ des cas ciblés, soit l'envoi de courriels aux pharmaciens, aux médecins et aux infirmières, souvent précédé d'une consultation des pharmaciens dans les équipes cliniques touchées et de la tenue d'une ou de plusieurs réunions avec les cliniciens concernés. En termes de charge de travail et de complexité, la difficulté ne réside pas dans la rédaction de courriels, mais bien dans la détermination de toutes les actions requises, leur coordination et leur implantation. Plusieurs actions décrites semblent à priori uniquement techniques (p. ex. création de nouvelles fiches de produits dans un logiciel, révision de quotas, ajout d'une alerte dans un logiciel, modification d'un processus de préparation par pompe). Dans un département de pharmacie, de nombreux logiciels sont utilisés pour la gestion des approvisionnements, pour le dossier clinique informatisé, pour le fonctionnement de différents automates, pour les armoires automatisées, etc. Chaque modification faite à la réserve des produits se répercute dans plusieurs systèmes. Un seul changement de produit peut nécessiter des heures de travail. Certains médicaments sont plus dangereux à manipuler que d'autres et si tous ces changements sont mal communiqués au personnel, ils peuvent entraîner des risques pour les patients. Il est souvent difficile de joindre tous les membres des équipes par un simple courriel. Dans certains cas ciblés, il faut augmenter le nombre de rencontres et de réunions pour mieux diffuser l'information au sujet de la rupture de stocks et des mesures prises pour en réduire les effets néfastes.

Fait rassurant, les dix pénuries ciblées dans notre enquête ont mené à un report d'activités cliniques chez $11 \%$ des répondants et un seul événement indésirable a été noté au cours de l’année de létude. Bien que ces données soient basées sur la mémoire des répondants, toutes les actions concertées menées par les pharmaciens hospitaliers ont porté des fruits, et les conséquences de ces pénuries sont négligeables pour les patients traités dans ces établissements de santé.

Enfin, les répondants étaient invités à faire part de leur degré d'accord à trois énoncés entourant la gestion des pénuries de médicaments. Tous s'accordent pour dire que les équipes de gestion des départements de pharmacie sont les mieux placées pour planifier les besoins en médicaments et tous les médicaments jugés importants pour la pratique devraient être stockés pour au moins 90 jours dans les réserves de l'établissement. Ceci contrevient aux pratiques financières et de gestion, qui visent une réserve minimale et un roulement des stocks élevé. Dans l'enquête canadienne sur la pharmacie hospitalière de 2016-2017, le taux moyen de roulement des stocks de médicaments est de 9,7 à léchelle du Canada et de 11,2 au Québec ${ }^{14}$. En favorisant des stocks de médicaments pour une période d’au moins 90 jours, le taux de roulement devra diminuer de façon significative et des espaces supplémentaires devront être consentis aux départements de pharmacie. De plus, les répondants notent qu'il est essentiel de préserver le rôle et le pouvoir d’achat des groupes d'approvisionnement en commun au Québec pour assurer la sécurité des stocks de médicaments en établissement de santé. Les ententes de partenariat négociées par l'Alliance pharmaceutique pancanadienne sont signées par les autorités provinciales et ceci contribue à fragmenter le pouvoir de négociation et le rapport de forces des GAC. Tous les répondants considèrent que le rôle et le pouvoir d'achat des GAC sont des éléments cruciaux pour la gestion des pénuries de médicaments. Enfin, neuf répondants sur dix considèrent que la baisse forcée des prix des médicaments génériques nest pas étrangère à la réduction des marges des stocks des fabricants de médicaments génériques au Canada. Les pharmaciens hospitaliers peuvent stocker davantage de produits, pour autant que ces médicaments demeurent disponibles sur le marché canadien. Une réflexion politique devrait s’engager sur les conditions permettant de préserver une disponibilité suffisante de médicaments sur le territoire canadien.

Cette étude comporte des limites. Létude ne cible qu'un GAC ; ce serait peut-être intéressant de répéter létude à plus grande échelle au Canada. Toutefois, les pénuries de médicaments sont le plus souvent nationales et non locales. Il est toutefois possible que les pratiques de gestion varient d'une 
région à l'autre. Létude ne cible que dix médicaments. Étant donné les centaines de produits en pénurie chaque année, il est possible que les actions à entreprendre diffèrent selon la sélection des produits utilisés pour une telle enquête. En outre, les répondants ont été invités à décrire et à quantifier les actions effectuées au cours de la dernière année. Un biais de mémoire est possible, bien que les données des répondants convergent.

\section{CONCLUSION}

Cette étude descriptive présente une analyse qualitative originale de la gestion des pénuries des médicaments et des actions mises en place en établissement de santé. L'étude met en évidence la variété et la complexité des actions requises pour assurer une prestation sécuritaire de soins. Les pénuries ont un impact sérieux sur le travail des pharmaciens hospitaliers et 25 actions pharmaceutiques peuvent être envisagées afin de limiter les conséquences des pénuries de médicaments.

\section{Références}

1. Pénuries de médicaments. Association des pharmaciens du Canada; [page consultée le 24 décembre 2019]. [en ligne] : https://www.pharmacists.ca/representation/enjeux/penuries-de-medicaments/

2. APhCpénuries de médicaments - sondage menéauprès de 1500 résidents canadiens. Asssociation des pharmaciens du Canada; [page consultée le 24 décembre 2019]. [en ligne] : https://www.pharmacists.ca/ cpha-fr/assets/File/CPhA-on-the-issues/DrugShortages_AbacusSurvey _November2018.pdf

3. Sondage 2018 de l'APhCsurlespénuries et rappels de médicaments. Association des pharmaciens du Canada; novembre 2018 [page consultée le 24 décembre 2019]. [en ligne] : https://www.pharmacists.ca/cpha-fr/ assets/File/CPhA-on-the-issues/CPhADrugShortagesandRecallsSurvey2018.pdf

4. Baumer AM, Clark AM, Witmer DR, Geize SB, Vermeulen LC, Deffenbaugh JH. National survey of the impact of drug shortages in acute care hospitals. Am J Health Syst Pharm. 2004;61(19):2015-22.

5. Gagnon MA. Drug shortages: searching for a cure. Health Policy. 2012; 7(4):10-7.

6. Videau M, Lebel D, Bussières JF. Drug shortages in Canada: data for 2016-2017 and perspectives on the problem. Ann Pharm Fr. 2019;77(3): 205-11.

7. Videau M, Chemali L, Stucki C, Saavedra-Mitjans M, Largana S, Guerin
A, et al. Drug shortages: cross-sectional comparison between France, Belgium, Spain, Switzerland and Canada. Can J Hosp Pharm. 2019; 72(1):7-15.

8. Rousseau A, Rinaldi F, Dubois S, Lebel D, Bussières JF. Pénuries de médicaments : 10 ans de recul au Canada. Pharmactuel. 2016;49(2):122-7.

9. Shaban H, Maurer C, Willborn RJ. Impact of drug shortages on patient safety and pharmacy operation costs. Fed Pract. 2018;35(1):24-31

10. Claus B, Pauwels K, Baert M, Depoorter J, De Weerdt E, Boussery K, et al. Indisponibilité des médicaments dans les hôpitaux : gestion, causes et impacts sur le budget. J Pharm Belg. 2015;(1):24-34.

11. De Weerdt E, De Rijdt T, Simoens S, Casteels M, Huys I. Time spent by Belgian hospital pharmacists on supply disruptions and drug shortages: an exploratory study. PLoS One. 2017;12(3):e0174556.

12. Pauwels K, Simoens S, Casteels M, Huys I. Insights into European drug shortages: a survey of hospital pharmacists. PLoS One. 2015; 10(3):e0119322.

13. Miljković N, Gibbons N, Batista A, Fitzpatrick RW, Underhill J, Horák P. Results of EAHP's 2018 survey on medicines shortages. Eur J Hosp Pharm. 2019;26(2):60-5.

14. MacNair K, Mills A. Drug distribution. In: Hospital pharmacy in Canada report 2016/17. Hospital Pharmacy in Canada Editorial Board; 2018 [page consultée le 24 décembre 2019]. [en ligne] : https://www. cshp.ca/sites/default/files/HPC\%20Survey/Report\%202018.pdf

Marine Floutier est assistante de recherche, Unité de recherche en pratique pharmaceutique et Département de pharmacie, CHU Sainte-Justine, Montréal (Québec). Elle est également étudiante en pharmacie (candidate pour le degré Pharm. D.) à la Faculté des Sciences Pharmaceutiques et Biologiques de Marseille, Marseille, France.

Suzanne Atkinson, B. Pharm., M. Sc., est chef-adjointe aux services pharmaceutiques, Unité de recherche en pratique pharmaceutique et Département de pharmacie, CHU Sainte-Justine, Montréal (Québec).

Stéphane Roux, B. Pharm., M. Sc., est chef-adjoint au Département de pharmacie, Centre hospitalier de l'Université de Montréal, Montréal (Québec).

Jean-François Bussières, B. Pharm., M. Sc., MBA, FCSHP, FOPQ, est directeur, Unité de recherche en pratique pharmaceutique et Département de pharmacie, CHU Sainte-Justine, et professeur titulaire de clinique, Faculté de pharmacie, Université de Montréal, Montréal (Québec).

Conflits d'intérêts : Aucune déclaration.

Adresse de correspondance :

Marine Floutier

CHU Sainte-Justine

3175, chemin de la Côte Sainte-Catherine

Montréal QC H3T 1C5

Courriel : marine.floutier@gmail.com

Financement : Aucun reçu. 